\title{
A Note on Asymptotic Evaluation of Some Hankel Transforms
}

\author{
By C. L. Frenzen* and R. Wong**
}

\begin{abstract}
Asymptotic behavior of the integral

$$
I_{f}(w)=\int_{0}^{\infty} e^{-x^{2}} J_{0}(w x) f\left(x^{2}\right) x d x
$$

is investigated, where $J_{0}(x)$ is the Bessel function of the first kind and $w$ is a large positive parameter. It is shown that $I_{f}(w)$ decays exponentially like $e^{-\gamma \boldsymbol{k}^{2}}, \gamma>0$, when $f(z)$ is an entire function subject to a suitable growth condition. A complete asymptotic expansion is obtained when $f(z)$ is a meromorphic function satisfying the same growth condition. Similar results are given when $f(z)$ has some specific branch point singularities.
\end{abstract}

1. Introduction. Recently, B. Gabutti proposed to us the problem of finding asymptotic expansions for integrals of the form

$$
I_{f}(w)=\int_{0}^{\infty} e^{-x^{2}} J_{0}(w x) f\left(x^{2}\right) x d x,
$$

where $J_{0}(x)$ is the Bessel function of order zero and $f(x)$ is a continuous function in $(0, \infty)$. According to Gabutti, integrals of this type occur in some problems of high-energy nuclear physics; see [2], [3]. Numerical evaluation of these integrals has been carried out by Gabutti himself [1], [2].

Clearly, the integral $I_{f}(w)$ is a special case of the Hankel transform defined by

$$
H(w)=\int_{0}^{\infty} h(x) J_{0}(w x) d x,
$$

and a considerable amount of work [4], [7], [9], [10], [11] has been done on the asymptotic evaluation of this transform. The major result is that if $h(x)$ has a power series expansion at $x=0$, then the asymptotic expansion of $H(w)$, as $w \rightarrow+\infty$, can be formally obtained by termwise integration (in some generalized sense). However, if one applies this result to the odd function $h(x)=e^{-x^{2}} f\left(x^{2}\right) x$, one obtains an asymptotic expansion whose coefficients are all zero. The same phenomenon also arises with Fourier integrals; see Olver [6, pp. 78-79]. This of course prompts one to suspect that the integral $I_{f}(w)$ may be exponentially decaying. That this suspicion should be sustained will be the purpose of this note. More specifically, we shall show

Received August 22, 1983; revised June 12, 1984 and November 2, 1984.

1980 Mathematics Subject Classification. Primary 41A60; Secondary 44A15.

Key words and phrases. Asymptotic expansion, Hankel transform, Bessel functions, Laplace's method.

${ }^{*}$ Current address: Mathematics Department, Southern Methodist University, Dallas, Texas 75275.

**The work of this author was done while he was on leave at the University of British Columbia during 1982-1983, and was partially supported by the Natural Sciences and Engineering Research Council of Canada under Grant A-7359. This author is an I. W. Killam Research Fellow, 1982-1984. 
that if $f(z)$ is an entire function satisfying a certain growth condition, then $I_{f}(w)$ decays like $e^{-\gamma w^{2}}$ for some $\gamma>0$. Moreover, we shall derive infinite asymptotic expansions for $I_{f}(w)$, when $f(z)$ is a meromorphic function satisfying the same growth condition. Our method is similar to that of Olver, namely, a shift of the integration path parallel to itself in the complex plane. Similar results are also obtained, when $f(z)$ is one of the following elementary functions: $z^{n} /(b+z)^{\nu}$, $\log (b+z)$ and $\log z$, where $b$ and $\nu$ are positive numbers and $n$ is a nonnegative integer.

2. The Case of Entire Functions. Let $\eta \geqslant 0$ and $\xi<1$, and let $f(z)$ be an entire function satisfying

$$
|f(z)| \leqslant M e^{\xi \operatorname{Re} z+\eta|\operatorname{Im} z|}, \quad M>0,
$$

for all sufficiently large $|z|$. Define

$$
\alpha=1-\xi+\eta^{2} /(1-\xi)
$$

THEOREM 1. Under the above condition, we have

$$
I_{f}(w)=O\left(e^{-w^{2} / 4 \alpha}\right) \quad \text { as } w \rightarrow+\infty .
$$

Proof. Let $m=1$ and $\nu$ tend to zero in Eq. (5) on p. 75 of [8] to obtain

$$
H_{0}^{(1)}\left(z e^{i \pi}\right)=H_{0}^{(1)}(z)-2 J_{0}(z) .
$$

Note that the Hankel function $H_{0}^{(1)}(z)$ has a logarithmic branch point at $z=0$. By taking a branch cut along the negative imaginary axis, it can be shown that

$$
I_{f}(w)=\frac{1}{2} \int_{-\infty}^{\infty} e^{-x^{2}} H_{0}^{(1)}(w x) f\left(x^{2}\right) x d x,
$$

where the branch of $H_{0}^{(1)}(z)$ is determined by taking the branch of $\log z$ which is real for positive $z$. Now, consider the contour integral

$$
C_{R}(w)=\frac{1}{2} \int_{\Gamma} e^{-z^{2}} H_{0}^{(1)}(w z) f\left(z^{2}\right) z d z
$$

where the path $\Gamma$, traversed in the positive direction, is the boundary of the rectangle whose vertices are at $(-R, 0),(R, 0),(R, w / 2 \alpha)$ and $(-R, w / 2 \alpha)$; here $R>0$ and $\alpha$ is given in (2.2). With the cut along the negative imaginary axis, by Cauchy's theorem, we have

$$
C_{R}(w)=0 \text { for all } R>0 .
$$

Letting $R \rightarrow+\infty$, the contribution from the portion of $\Gamma$ parallel to the imaginary axis tends to zero by condition (2.1). The remaining horizontal pieces of $(2.5)$ reduce to

$$
I_{f}(w)=\frac{1}{2} \int_{-\infty+i w / 2 \alpha}^{\infty+i w / 2 \alpha} e^{-z^{2}} H_{0}^{(1)}(w z) f\left(z^{2}\right) z d z .
$$

In view of the well-known result $[8$, p. 219]

$$
\left|H_{0}^{(1)}(z)\right| \leqslant\left|\left(\frac{2}{\pi z}\right)^{1 / 2} e^{i z}\right|, \quad 0 \leqslant \arg z \leqslant \pi,
$$


the integral on the right-hand side of (2.7) is easily seen to be dominated by

$$
\begin{aligned}
\frac{M}{\sqrt{2 \pi w}} & \exp \left\{w^{2}\left(\frac{1-\xi}{4 \alpha^{2}}-\frac{1}{2 \alpha}\right)\right\} \\
& \times \int_{-\infty}^{\infty} e^{-(1-\xi) x^{2}+\eta(w / \alpha)|x|}\left(x^{2}+\frac{w^{2}}{4 \alpha^{2}}\right)^{1 / 4} d x
\end{aligned}
$$

Since

$$
\left(x^{2}+\frac{w^{2}}{4 \alpha^{2}}\right)^{1 / 4} \leqslant\left(\frac{2 \alpha}{w}\right)^{3 / 2}\left(x^{2}+\frac{w^{2}}{4 \alpha^{2}}\right),
$$

upon splitting the last integral at $x=0$, completing the square in the exponents of each of the two integrals and recombining them, we can show that the integral in (2.9) is dominated by

$$
2\left(\frac{2 \alpha}{w}\right)^{3 / 2} \exp \left\{\frac{\eta^{2}(w / \alpha)^{2}}{4(1-\xi)}\right\} \int_{0}^{\infty}\left(x^{2}+\frac{w^{2}}{4 \alpha^{2}}\right) \exp \left\{-\left(\sqrt{1-\xi} x-\frac{\eta w / \alpha}{2 \sqrt{1-\xi}}\right)^{2}\right\} d x
$$

Making the change of variable

$$
u=\sqrt{1-\xi} x-\frac{\eta(w / \alpha)}{2 \sqrt{1-\xi}}
$$

the above integral becomes, by using (2.2),

$$
\frac{1}{\sqrt{1-\xi}} \int_{-\eta(w / \alpha) /(2 \sqrt{1-\xi})}^{\infty} e^{-u^{2}}\left[\frac{1}{1-\xi} u^{2}+\frac{\eta(w / \alpha)}{(1-\xi)^{3 / 2}} u+\frac{w^{2}}{4 \alpha(1-\xi)}\right] d u
$$

which is clearly dominated by

$$
\begin{aligned}
\frac{1}{\sqrt{1-\xi}}\left\{\frac{1}{1-\xi} \int_{-\infty}^{\infty} u^{2} e^{-u^{2}} d u+\frac{\eta(w / \alpha)}{(1-\xi)^{3 / 2}} \int_{-\infty}^{\infty}|u| e^{-u^{2}} d u\right. & \\
& \left.+\frac{w^{2}}{4 \alpha(1-\xi)} \int_{-\infty}^{\infty} e^{-u^{2}} d u\right\}
\end{aligned}
$$

A combination of the above results shows

$$
\left|I_{f}(w)\right| \leqslant M^{\prime} \exp \left(-\frac{w^{2}}{4 \alpha}\right)
$$

where

$$
M^{\prime}=\frac{M \alpha^{1 / 2}}{(1-\xi)^{3 / 2}}\left[1+\frac{4 \eta}{\sqrt{\pi(1-\xi)}} \frac{1}{w}+\frac{2 \alpha}{w^{2}}\right]
$$

This completes the proof of the theorem.

Condition (2.1) may be slightly weakened. For instance, we may replace it by

$$
|f(z)| \leqslant M|z|^{n} e^{\xi \operatorname{Re} z+\eta|\operatorname{Im} z|}
$$

for all large $|z|$, where $\eta>0, \xi<1$ and $n$ is a positive integer. The order estimate for $I_{f}(w)$ is then given by $O\left(w^{2 n} e^{-w^{2} / 4 \alpha}\right)$.

Note that the above method cannot be extended to the more general integral

$$
\int_{0}^{\infty} e^{-x^{2}} J_{\nu}(w x) f\left(x^{2}\right) x d x
$$


unless $\nu$ is an even integer. This is due to the fact that the identity

$$
H_{\nu}^{(1)}\left(t e^{i \pi}\right)=H_{\nu}^{(1)}(t)-2 J_{\nu}(t)
$$

holds only when $\nu=2 m$, where $m$ is an integer; see [8, p. 75, Eq. (5)]. In this case the proof proceeds as before. If $\nu$ is not an even integer, then the term $H_{\nu}^{(1)}\left(t e^{\pi i}\right)$ in the above equation is multiplied by the factor $e^{\nu \pi i}$, in which case the above integral on $(0, \infty)$ cannot be rewritten in the form of an integral on $(-\infty, \infty)$ corresponding to the one given in (2.4).

As an example of Theorem 1, we consider the integral

$$
I(w)=\int_{0}^{\infty} e^{-x^{2}} J_{0}(w x) x \sin x^{2} d x .
$$

Since $f(z)=\sin z$ is entire and bounded by $e^{|\operatorname{Im} z|}$, we have $\xi=0$ and $\eta=+1$. Equation (2.2) then gives $\alpha=2$. From Theorem 1, it now follows that

$$
I(w)=O\left(e^{-u^{2} / 8}\right) .
$$

The integral $I(w)$ was used by Gabutti [1], [2] to illustrate his numerical procedures; however, no mention was made of the exact evaluation

$$
I(w)=\frac{1}{4} e^{-w^{2} / 8}\left(\cos \frac{w^{2}}{8}-\sin \frac{w^{2}}{8}\right) .
$$

The result (2.12) follows immediately from the well-known identity [8, p. 393; 5, p. 8]

$$
\int_{0}^{\infty} x e^{-\lambda x^{2}} J_{0}(w x) d x=\frac{1}{2 \lambda} e^{-u^{2} / 4 \lambda} ; \quad \operatorname{Re} \lambda>0,
$$

by taking the imaginary part of both sides with $\lambda=1-i$.

3. The Meromorphic Case. We first note that if $f(z)$ is a meromorphic function with a finite number, say, $m$ of poles located away from the nonnegative real axis, then $f\left(z^{2}\right)$ is a meromorphic function with $2 m$ poles located away from the real axis, with $m$ poles in the upper half-plane and $m$ poles in the lower half-plane. Hence, for convenience, we may state the following useful result in terms of $f\left(z^{2}\right)$ and its poles in the upper half-plane.

THEOREM 2. Let $f(z)$ be a meromorphic function such that $f\left(z^{2}\right)$ has a finite number of poles located in the upper half-plane, say, at $a_{1}, \ldots, a_{m}$, and let $f(z)$ satisfy the growth condition in (2.1). Then, as $w \rightarrow+\infty$,

$$
I_{f}(w)=\pi i \sum_{j=1}^{m} \operatorname{Res}\left\{e^{-z^{2}} H_{0}^{(1)}(w z) f\left(z^{2}\right) z ; a_{j}\right\}+O\left(e^{-w^{2} / 4 \alpha}\right),
$$

$\alpha$ being the same as in Theorem 1 .

Proof. The proof is identical to that of Theorem 1, except that as we deform the contours of $C_{R}(w)$, we pick up the residues from the poles of $f\left(z^{2}\right)$.

An immediate consequence of (3.1) is that as $w \rightarrow+\infty$,

$$
I_{f}(w)=O\left(e^{-\delta w}\right)
$$

where $\delta=\min \left\{\operatorname{Im} a_{i}: i=1, \ldots, m\right\}$. This follows from the fact that $H_{0}^{(1)}(w z)$ decays exponentially like $e^{-w \operatorname{Im} z}$ in the upper half-plane; see Eq. (2.8). 
As an example of Theorem 2, we consider the integral

$$
I^{(3)}(w)=\int_{0}^{\infty} e^{-x^{2}} J_{0}(w x) \frac{x}{1+x^{2}} d x .
$$

Since $f\left(z^{2}\right)=\left(1+z^{2}\right)^{-1}$ has a simple pole in the upper half-plane at $z=i$, Theorem 2 gives

$$
I^{(3)}(w)=e K_{0}(w)+O\left(e^{-w^{2} / 4}\right),
$$

where $K_{0}(w)$ is the modified Bessel function. The well-known asymptotic expansion of $K_{0}(w)$ then yields

$$
I^{(3)}(w) \sim \sqrt{\frac{\pi}{2}} e^{-w+1}\left[w^{-1 / 2}-\frac{1}{8} w^{-3 / 2}+\frac{9}{128} w^{-5 / 2}+\cdots\right] .
$$

An alternative method of deriving (3.3) is to introduce a parameter into the exponential function in (3.2) and to obtain a first-order linear nonhomogeneous ordinary differential equation with the parameter as the independent variable. This equation can be solved in closed form and the result in (3.3) can be recovered by setting the parameter equal to one.

4. Algebraic Singularities. Let $\nu$ be real, $n=1,2, \ldots$, and consider the integral

$$
I^{(4)}(w)=\int_{0}^{\infty} e^{-x^{2}} J_{0}(w x) \frac{x^{2 n+1}}{\left(a^{2}+x^{2}\right)^{\nu}} d x,
$$

where $a$ is a positive number. The case when $\nu$ is an integer is covered by Theorems 1 and 2. However, if $\nu$ is not an integer, then we must proceed in a different manner. Let us first assume that $\nu$ is positive.

Inserting the identity

$$
\frac{1}{\left(a^{2}+x^{2}\right)^{\nu}}=\frac{1}{\Gamma(\nu)} \int_{0}^{\infty} t^{\nu-1} e^{-\left(a^{2}+x^{2}\right) t} d t
$$

in (4.1) and reversing the order of integration, we have from [8, p. 394 (3)]

$$
I^{(4)}(w)=\frac{n !}{2 \Gamma(\nu)} \sum_{k=0}^{n}\left(\begin{array}{l}
n \\
k
\end{array}\right) \frac{(-1)^{k} w^{2 k}}{4^{k} k !} \Phi_{n+k}(w),
$$

where

$$
\Phi_{m}(w)=\int_{0}^{\infty} \frac{t^{\nu-1}}{(1+t)^{m+1}} \exp \left\{-a^{2} t-\frac{w^{2}}{4(1+t)}\right\} d t .
$$

From a numerical point of view, the integral $\Phi_{m}(w)$ is easier to handle than the integral $I^{(4)}(w)$, since the integrand in (4.4) does not involve the Bessel function and is positive. To derive an asymptotic expansion for $\Phi_{m}(w)$, we prove the following general theorem.

THEOREM 3. Let $\nu$ and $c$ be positive, let $f(t)$ be a continuous function in $(0, \infty)$ satisfying the following conditions:

(i) $f(t)=O\left(t^{-\delta}\right)$, as $t \rightarrow 0^{+}$, for some $\delta \geqslant 0$;

(ii) $f(t)=O\left(e^{\rho t}\right)$, as $t \rightarrow \infty$, for some $\rho>0$;

(iii) $f(t)$ is analytic in a neighborhood of $t=1$. 
Put

$$
F(\lambda)=\int_{c / \lambda}^{\infty}\left(t-\frac{c}{\lambda}\right)^{\nu-1} f(t) \exp \left\{-\lambda\left(t+\frac{1}{t}\right)\right\} d t
$$

Then, as $\lambda \rightarrow+\infty$, we have the generalized asymptotic expansion $[6, p .25]$

$$
F(\lambda) \sim e^{-2 \lambda}\left[\sum_{n=0}^{\infty} c_{n, \nu}(\lambda) \Gamma\left(n+\frac{1}{2}\right) \lambda^{-n-1 / 2} ;\left\{\lambda^{-n-1 / 2}\right\}\right],
$$

where each $c_{n, \nu}(\lambda)$ is a bounded continuous function of $\lambda$ in $(0, \infty)$ and is bounded away from zero for all large values of $\lambda$.

Proof. Our argument will be based on the well-known method of Laplace [6, pp. 85-86], and, without loss of generality, we shall take $\lambda>2 c$. Splitting the interval of integration at $t=\frac{1}{2}$ and $t=1$, we have

$$
F(\lambda)=E(\lambda)+F_{1}(\lambda)+F_{2}(\lambda)
$$

where $E(\lambda), F_{1}(\lambda)$ and $F_{2}(\lambda)$ correspond, respectively, to the intervals $(c / \lambda, 1 / 2)$, $(1 / 2,1)$ and $(1, \infty)$. Since the function $t+t^{-1}$ is decreasing in $(0,1)$, the integral $E(\lambda)$ is bounded by

$$
\exp \left\{-\lambda\left(\frac{c}{\lambda}+\frac{\lambda}{c}\right)\right\} \int_{c / \lambda}^{1 / 2}\left(t-\frac{c}{\lambda}\right)^{\nu-1}|f(t)| d t .
$$

By condition (i), it is easily seen that the last integral is $O\left(\lambda^{\delta}\right)$. Hence, we have the order estimate

$$
E(\lambda)=O\left(\lambda^{\delta} e^{-\lambda^{2} / c}\right)
$$

Anticipating the final result, we may drop this term and write

$$
F(\lambda) \approx F_{1}(\lambda)+F_{2}(\lambda) \text { as } \lambda \rightarrow+\infty .
$$

In the integrals $F_{1}(\lambda)$ and $F_{2}(\lambda)$, we shall make the change of variables

$$
t+\frac{1}{t}-2=\tau
$$

The function on the left is strictly decreasing in $\frac{1}{2}<t<1$ and strictly increasing in $t>1$, and maps the interval $1<t<\infty$ onto $0<\tau<\infty$ and $\frac{1}{2}<t<1$ onto $\frac{1}{2}>\tau>0$. From these monotonicity properties, it follows that the transformation (4.9) is invertible. Denote the inverse by

$$
t=t_{ \pm}(\tau)
$$

where the plus sign is to be used for $\tau$ increasing (i.e., in $F_{2}(\lambda)$ ) and the minus sign is to be used for $\tau$ decreasing (i.e., in $F_{1}(\lambda)$ ). Upon this change of variables, the integrals $F_{1}(\lambda)$ and $F_{2}(\lambda)$ become

$$
F_{1}(\lambda)=-e^{-2 \lambda} \int_{0}^{\infty} f_{-}(\tau ; \lambda) e^{-\lambda \tau} d \tau \text { and } F_{2}(\lambda)=e^{-2 \lambda} \int_{0}^{\infty} f_{+}(\tau ; \lambda) e^{-\lambda \tau} d \tau,
$$

where

$$
f_{ \pm}(\tau ; \lambda)=\left(t_{ \pm}(\tau)-\frac{c}{\lambda}\right)^{\nu-1} f\left(t_{ \pm}(\tau)\right) \frac{d t_{ \pm}}{d \tau}
$$

and $f_{-}(\tau ; \lambda)$ is defined to be zero for $\tau \geqslant \frac{1}{2}$. Put

$$
f(\tau ; \lambda) \equiv f_{+}(\tau ; \lambda)-f_{-}(\tau ; \lambda) .
$$


Then

$$
F_{1}(\lambda)+F_{2}(\lambda)=e^{-2 \lambda} \int_{0}^{\infty} f(\tau ; \lambda) e^{-\lambda \tau} d \tau
$$

We shall next show that in a neighborhood of $\tau=0$, we have

$$
f(\tau ; \lambda)=\sum_{n=0}^{\infty} c_{n, \nu}(\lambda) \tau^{n-1 / 2},
$$

where the coefficients $c_{n, \nu}(\lambda)$ are exactly those given in the final result (4.6), which will now follow immediately from (4.8), (4.12) and (a modification of) Watson's lemma [6, p. 71].

To prove (4.13), we note that Eq. (4.9) gives

$$
t_{ \pm}(\tau)=\frac{1}{2}\left[2+\tau \pm \sqrt{\tau^{2}+4 \tau}\right] .
$$

For $0<\tau<4$, the right-hand side of this equation can be represented by the power series

$$
\sigma_{ \pm}(\sqrt{\tau})=1+\frac{( \pm \sqrt{\tau})^{2}}{2}+2 \sum_{k=0}^{\infty}\left(\begin{array}{c}
1 / 2 \\
k
\end{array}\right)\left(\frac{ \pm \sqrt{\tau}}{2}\right)^{2 k+1} .
$$

Observe that for $0<\tau<4$, we have

$$
t_{ \pm}(\tau)=\sigma_{ \pm}(\sqrt{\tau})
$$

and

$$
\sigma_{+}(\sqrt{\tau})=\sigma_{-}(-\sqrt{\tau}) .
$$

If $F_{ \pm}(\sqrt{\tau} ; \lambda)$ denotes the quantities

$$
\left(\sigma_{ \pm}(\sqrt{\tau})-\frac{c}{\lambda}\right)^{\nu-1} f\left(\sigma_{ \pm}(\sqrt{\tau})\right) \frac{d}{d \tau} \sigma_{ \pm}(\sqrt{\tau}),
$$

then from (4.15) and (4.16) it follows that for $0<\tau<4$,

$$
f_{ \pm}(\tau ; \lambda)=F_{ \pm}(\sqrt{\tau} ; \lambda)
$$

and

$$
F_{+}(\sqrt{\tau} ; \lambda)=F_{-}(-\sqrt{\tau} ; \lambda) .
$$

Since $(t-c / \lambda)^{\nu-1} f(t)$ is analytic in a neighborhood of $t=1$, it is clear from (4.14) and (4.18) that there exists a positive number $\delta$ such that for $0<\tau<\delta, F_{ \pm}(\sqrt{\tau} ; \lambda)$ has a convergent expansion of the form

$$
F_{ \pm}(\sqrt{\tau} ; \lambda)=\sum_{n=0}^{\infty}( \pm 1)^{n-1} a_{n, \nu}(\lambda) \tau^{(n-1) / 2}
$$

where the coefficients $a_{n, \nu}(\lambda)$ have the same properties as those $c_{n, \nu}(\lambda)$ given in (4.6). Relations (4.11) and (4.17) then give

$$
f(\tau ; \lambda)=2 \sum_{n=0}^{\infty} a_{2 n, \nu}(\lambda) \tau^{n-1 / 2} .
$$

With $c_{n, \nu}(\lambda)=2 a_{2 n, \nu}(\lambda)$, this establishes the result in (4.13) and hence the theorem. 
The coefficients $c_{n, \nu}(\lambda)$ can be calculated explicitly, and, for convenience of application, we give the first three:

$$
\begin{gathered}
c_{0, \nu}(\lambda)=\left(1-\frac{c}{\lambda}\right)^{\nu-1} f(1), \\
c_{1, \nu}(\lambda)=\sum_{k=0}^{2}\left(1-\frac{c}{\lambda}\right)^{\nu-1-k}\left(\begin{array}{c}
\nu-1 \\
k
\end{array}\right) \sum_{s=0}^{2-k} b_{k s} \frac{f^{(s)}(1)}{s !},
\end{gathered}
$$

where

$$
\begin{array}{ll}
b_{20}=1, & \\
b_{10}=\frac{3}{2}, & b_{11}=1 \\
b_{00}=\frac{3}{8}, & b_{01}=\frac{3}{2}, \quad b_{02}=1,
\end{array}
$$

and

$$
c_{2, \nu}(\lambda)=\sum_{k=0}^{4}\left(1-\frac{c}{\lambda}\right)^{\nu-1-k}\left(\begin{array}{c}
\nu-1 \\
k
\end{array}\right) \sum_{s=0}^{4-k} a_{k s} \frac{f^{(s)}(1)}{s !}
$$

where

$$
\begin{array}{llll}
a_{40} & =1, \\
a_{30} & =\frac{5}{2}, & a_{31}=1, & \\
a_{20}=\frac{15}{8}, & a_{21}=\frac{5}{2}, & a_{22}=1, & \\
a_{10}=\frac{5}{16}, & a_{11}=\frac{15}{8}, & a_{12}=\frac{5}{2}, & a_{13}=1, \\
a_{00}=-\frac{5}{128}, & a_{01}=\frac{5}{16}, & a_{02}=\frac{15}{8}, & a_{03}=\frac{5}{2}, \quad a_{04}=1 .
\end{array}
$$

Also, it is easily seen that each $c_{n, \nu}(\lambda)$ can be expanded into an asymptotic expansion in powers of $1 / \lambda$, and that the generalized asymptotic expansion (4.6) can be rewritten in the Poincaré form

$$
F(\lambda) \sim e^{-2 \lambda} \sum_{n=0}^{\infty} d_{n}(\nu) \lambda^{-n-1 / 2},
$$

where the coefficients $d_{n}(\nu)$ are independent of $\lambda$. However, in many circumstances, we find it more convenient to apply the result in (4.6) directly.

Returning to (4.4), we make the substitution

$$
1+t=\frac{w}{2 a} \tau .
$$

The function $\Phi_{m}(w)$ then becomes

$$
\Phi_{m}(w)=e^{a^{2}}\left(\frac{w}{2 a}\right)^{\nu-m-1} \int_{2 a / w}^{\infty}\left(\tau-\frac{2 a}{w}\right)^{\nu-1} \tau^{-m-1} \exp \left\{-\frac{a w}{2}\left(\tau+\frac{1}{\tau}\right)\right\} d \tau .
$$

The last integral is of the form (4.5), with $\lambda=a w / 2, c=a^{2}$ and $f(t)=t^{-m-1}$. Thus, by Theorem 3,

$$
\Phi_{m}(w) \sim\left(\frac{w}{2 a}\right)^{\nu-m-1} e^{-w a+a^{2}}\left[\alpha_{0}(w) w^{-1 / 2}+\alpha_{1}(w) w^{-3 / 2}+\cdots\right]
$$


The coefficients $\alpha_{n}(w)$ can be obtained from the expressions for $c_{n, \nu}(\lambda)$. In particular, we have

$$
\alpha_{0}(w)=\sqrt{\frac{2 \pi}{a}}\left(1-\frac{2 a}{w}\right)^{\nu-1} .
$$

Inserting the result (4.20) into (4.3) gives the asymptotic expansion of $I^{(4)}(w)$.

As a special case, we obtain

$$
\int_{0}^{\infty} e^{-x^{2}} J_{0}(w x) \frac{x}{\sqrt{1+x^{2}}} d x \sim e^{-w+1}\left[w^{-1}+w^{-2}+\frac{5}{2} w^{-3}+\cdots\right]
$$

To derive a similar result for the case $f\left(x^{2}\right)=\sqrt{1+x^{2}}$, we write

$$
\sqrt{1+x^{2}}=\frac{1}{\sqrt{1+x^{2}}}+\frac{x^{2}}{\sqrt{1+x^{2}}} \text {. }
$$

Both functions on the right-hand side are of the form considered in Eq. (4.1), and hence the above analysis applies. This device, of course, extends to the more general function $f\left(x^{2}\right)=\left(a^{2}+x^{2}\right)^{\mu}$, where $a$ and $\mu$ may be any positive numbers, thus removing the assumption $\nu>0$ imposed earlier.

5. The Logarithmic Case. A simple and tempting way to derive an asymptotic expansion for the integral

$$
I^{(5)}(w)=\int_{0}^{\infty} e^{-x^{2}} J_{0}(w x) \log \left(a^{2}+x^{2}\right) x d x
$$

is to differentiate the expansion

$$
\int_{0}^{\infty} e^{-x^{2} J_{0}(w x)} \frac{x}{\left(a^{2}+x^{2}\right)^{\nu}} d x \sim \frac{1}{2 \Gamma(v)} e^{-w a+a^{2}}\left(\frac{w}{2 a}\right)^{\nu-1} \sum_{n=0}^{\infty} b_{n}(\nu) w^{-n-1 / 2}
$$

(obtained from (4.3) and (4.20) with $n=m=0$ ) with respect to $\nu$ and then set $\nu=0$. However, this formal derivation is not easy to justify, and hence we shall proceed in a different manner. Our analysis here is essentially that given in Section 4, except for some minor modifications.

We first recall the identity

$$
\log \left(a^{2}+x^{2}\right)=-\gamma-\left(a^{2}+x^{2}\right) \int_{0}^{\infty}(\log t) e^{-\left(a^{2}+x^{2}\right) t} d t
$$

Inserting it into (5.1), we obtain

$$
I^{(5)}(w)=-\frac{\gamma}{2} e^{-w^{2} / 4}-\Psi(w),
$$

where

$$
\begin{array}{r}
\Psi(w)=\int_{0}^{\infty}(\log t)\left[\frac{a^{2}}{2(1+t)}+\frac{1}{2(1+t)^{2}}-\frac{w^{2}}{8(1+t)^{3}}\right] \\
\times \exp \left\{-a^{2} t-\frac{w^{2}}{4(1+t)}\right\} d t
\end{array}
$$

The substitution $1+t=(w / 2 a) \tau$ then gives

$$
e^{-a^{2}}\left(\frac{2 a}{w}\right) \Psi(w)=\log \left(\frac{w}{2 a}\right) \int_{2 a / w}^{\infty} G(\tau ; w) d \tau+\int_{2 a / w}^{\infty} \log \left(\tau-\frac{2 a}{w}\right) G(\tau ; w) d \tau,
$$


where

$$
G(\tau ; w)=\left(\frac{a^{3}}{w \tau}+\frac{2 a^{2}}{w^{2} \tau^{2}}-\frac{a^{3}}{w \tau^{3}}\right) \exp \left\{-\frac{w a}{2}\left(\tau+\frac{1}{\tau}\right)\right\} .
$$

By integration by parts, the first integral on the right can be evaluated exactly to be

$$
\frac{a}{w} e^{-a^{2}-w^{2} / 4} \text {. }
$$

To the second integral on the right, we can apply a variation of Theorem 3 . Disregarding terms of the order $e^{-w^{2} / 4}$, we arrive at the final result

$$
I^{(5)}(w) \sim-\sqrt{2 \pi a} e^{-w a+a^{2}}\left[e_{0} w^{-3 / 2}+e_{1} w^{-5 / 2}+e_{2} w^{-7 / 2}+\cdots\right],
$$

where $e_{0}=1, e_{1}=2 a+3 / 8 a, e_{2}=4 a^{2}+15 / 4-15 / 128 a^{2}$.

For completeness, we include a discussion of the case $f\left(x^{2}\right)=\log x^{2}$. Here, as we shall see, the integral $I_{f}(w)$ is not exponentially small, in contrast with the other results obtained thus far. This is probably due to the fact that the log function does not have a power series expansion at the origin. We also point out that since the coefficients $e_{n}$ in (5.2) depend on $a$ and are not continuous at $a=0$, the asymptotic expansion of the integral

$$
I^{(6)}(w)=\int_{0}^{\infty} e^{-x^{2}} J_{0}(w x)\left(\log x^{2}\right) x d x
$$

will not follow from (5.2) by letting $a$ tend to zero.

Replacing $J_{0}(w x)$ by its Maclaurin series and integrating term by term, one readily obtains

$$
\begin{aligned}
\int_{0}^{\infty} e^{-x^{2} J_{0}(w x) x^{\mu} d x} & =\frac{1}{2} \Gamma\left(\frac{\mu+1}{2}\right){ }_{1} F_{1}\left(\frac{\mu+1}{2} ; 1 ;-\frac{w^{2}}{4}\right) \\
& =\frac{1}{2} \Gamma\left(\frac{\mu+1}{2}\right) \exp \left(-\frac{w^{2}}{4}\right){ }_{1} F_{1}\left(\frac{1-\mu}{2} ; 1 ; \frac{w^{2}}{4}\right),
\end{aligned}
$$

where ${ }_{1} F_{1}(a ; c ; z)$ is the confluent hypergeometric function defined by

$$
{ }_{1} F_{1}(a ; c ; z)=\sum_{k=0}^{\infty} \frac{(a)_{k}}{(c)_{k}} \frac{z^{k}}{k !}, \quad|z|<\infty, c \neq 0,-1,-2, \ldots,
$$

and $(a)_{k}$ is the Pochhammer notation

$$
(a)_{0}=1, \quad(a)_{k}=a(a+1) \cdots(a+k-1), \quad k=1,2, \ldots
$$

The second equality in (5.4) is obtained by Kummer's transformation; see [8, p. 394]. It is easily verified that

$$
\frac{d}{d a}(a)_{k}=(a)_{k}[\psi(a+k)-\psi(a)] \text { and } \lim _{a \rightarrow 0}(a)_{k} \psi(a)=-(k-1) !
$$

$\psi$ being the logarithmic derivative of the $\Gamma$-function. Differentiating both sides of (5.4) with respect to $\mu$ and setting $\mu=1$, we obtain

$$
I^{(6)}(w)=-\frac{1}{8} \exp \left(-\frac{w^{2}}{4}\right)\left[\gamma+E^{*}\left(\frac{w^{2}}{4}\right)\right]
$$

where

$$
E^{*}(z)=\sum_{k=1}^{\infty} \frac{z^{k}}{k \cdot k !}
$$


The function $E^{*}(z)$ can be expressed in terms of the complementary exponential integral $\operatorname{Ein}(z)$, see [6], and it can be shown that as $z \rightarrow+\infty$,

$$
E^{*}(z) \sim e^{z} \sum_{n=0}^{\infty} \frac{n !}{z^{n+1}} .
$$

Coupling (5.5) and (5.6) gives

$$
I^{(6)}(w) \sim-\sum_{n=0}^{\infty} 2^{2 n+1} n ! w^{-2 n-2}
$$

as $w \rightarrow+\infty$. This result also follows from Theorem 2 in Soni [7]; note, in particular, Eq. (3.5) there.

6. Conclusion. Asymptotic behavior of the integral $I_{f}(w)$, given in (1.1), has been investigated for functions $f(z)$ which are entire or meromorphic and satisfy growth condition (2.1). For these two cases, it was shown that $I_{f}(w)=O\left(e^{-\gamma w^{2}}\right)$ and $I_{f}(w)=O\left(e^{-\delta w}\right)$, respectively, as $w \rightarrow+\infty$, where $\gamma$ and $\delta$ are positive numbers. In fact, explicit asymptotic expansions of $I_{f}(w)$ were obtained in the case of meromorphic functions. Several specific examples of branch point singularities were also considered. Our results seem to suggest that (i) if $f(z)$ has a Maclaurin expansion with finite positive radius of convergence, then $I_{f}(w)$ has an asymptotic expansion of the form

$$
e^{-w \beta} \sum_{n=0}^{\infty} C_{n} w^{-n / 2} \text { as } w \rightarrow+\infty,
$$

for some $\beta>0$, and (ii) if $f(z)$ does not have a Maclaurin expansion, then $I_{f}(w)$ decays only algebraically. When $f(z)$ is meromorphic, the expansion given in (i) follows from Theorem 2. To support the statement in (ii), we mention the following example in addition to the results in (5.4), when $\mu$ is not an odd positive integer, and (5.7). Let $f(z)=\sin \sqrt{z}$, and observe that Theorem 2 above does not apply to this function. Nevertheless, by Theorem 2 in [9], we have

$$
I_{f}(w) \sim-w^{-3}-\frac{21}{2} w^{-5}-\frac{1215}{8} w^{-7}+\cdots .
$$

Acknowledgment. We would like to thank the referees for a careful reading of this paper and some constructive suggestions.

\footnotetext{
Department of Mathematics

University of British Columbia

Vancouver, B.C., Canada V6T 1Y4

Department of Mathematics

University of Manitoba

Winnipeg, Manitoba, Canada R3T 2N2
}

1. B. Gabutti, "On high precision methods for computing integrals involving Bessel functions," Math. Comp., v. 33, 1979, pp. 1049-1057.

2. B. Gabutti \& B. MinetTi, "A new application of the discrete Laguerre polynomials in the numerical evaluation of the Hankel transform of a strongly decreasing even function," J. Comput. Phys., v. 42, 1981, pp. $277-287$.

3. R. J. Glauber, Lectures in Theoretical Physics, vol. 1, Interscience, New York, 1959.

4. R. A. Handelsman \& J. S. Lew, "Asymptotic expansion of a class of integral transforms with algebraically dominated kernels," J. Math. Anal. Appl., v. 35, 1971, pp. 405-433. 
5. F. Oberhettinger, Tables of Bessel Transforms, Springer, Berlin, 1972.

6. F. W. J. Olver, Asymptotics and Special Functions, Academic Press, New York, 1974.

7. K. SonI, "Asymptotic expansion of the Hankel transform with explicit remainder terms," Quart. Appl. Math., v. 40, 1982, pp. 1-14.

8. G. N. Watson, A Treatise on the Theory of Bessel Functions, 2nd ed., Cambridge Univ. Press., Cambridge, 1944.

9. R. Wong, "Error bounds for asymptotic expansions of Hankel transforms," SIAM J. Math. Anal., v. 7, 1976, pp. 799-808.

10. R. WONG, "Error bounds for asymptotic expansions of integrals," SIAM Rev., v. 22, 1980, pp. 401-435.

11. A. I. ZAYED, “Asymptotic expansions of integral transforms by using generalized functions," Trans. Amer. Math. Soc., v. 272, 1982, pp. 785-802. 